\title{
The Smart Campus Design and Implementation in Vocational College
}

\author{
Wei Wei \\ Wuhan City Vocational College, Wuhan, Hubei Province, China
}

Keywords: Vocational colleges, smart campus, design, implementation

\begin{abstract}
At present, with the continuous advancement of college information construction, many colleges and universities and enterprises have successively launched their own plans and strategies for information construction. This paper aims at the current construction of digital campus in higher vocational colleges and advances to the smart campus. The design and implementation of smart campus in higher vocational colleges was proposed to provide reference for the construction of smart campus in various universities.
\end{abstract}

\section{Introduction}

With the in-depth application of new technologies such as big data, mobile Internet, cloud computing, virtualization, and the Internet of Things, China's university informationization is rapidly entering the "smart campus" stage from the "digital campus" to realize intelligence. A school is a publicly-owned provincial vocational college. There are 98 multimedia classrooms, 6 computer classrooms, and 1 HD recording classroom in the two campuses. There are more than 9,000 full-time students and 23 administrative offices [1]. After years of informatization construction, there are now complete basic information facilities, but there are still outstanding problems such as network export shortages, insufficient capacity of data centers, lack of integration of business systems, and poor resource sharing mechanisms.

\section{Smart campus construction status and problems}

The construction of a smart campus must be based on the actual research and development of the university. Only in this way can we ensure good expansion and exuberant vitality. At present, the application system built in A School includes application systems such as enrollment management system, digital orientation system, dormitory management system, academic management system, electronic school leaving system, collaborative office system, and educational administration system. However, these systems are in data and applications [1]. All of them are mutually exclusive and do not share with each other, and each system has its own governance, lack of coordination, resulting in many data islands. In the era of big data, heterogeneous data types, widely existing data sources, and uneven data quality have brought new challenges to data integration.

\subsection{Inadequate information construction of education and teaching}

Whether in teaching management or scientific research, the level of application of vocational education information is generally low. Therefore, the low-level application of information technology cannot fully mobilize the enthusiasm of students to learn, cannot satisfy their craving for knowledge, and hinder their in-depth research and exploration of knowledge [1]. This is also one of the bottlenecks of the rapid development of the level of vocational education informatization.

At present, the total amount of quality digital education resources is insufficient, and an effective sharing mechanism has not yet been formed. Teachers and students cannot obtain information such as learning through the Internet, and they cannot obtain information on education policies and related management through the Internet. The quality of curriculum resources is relatively scarce. After the national backbone network of education informatization was established, the information resources on the network were seriously insufficient [2]. First, the information resources available for use were not abundant. More management information, less application resources, more teaching assistant 
resources, less scholarship resources, more public course resources, less specialized course resources, more specialized websites, fewer integrated websites, and second, manifested in sharable resources not much. Due to the current lack of an effective sharing mechanism, the interests of vocational education developers are not guaranteed, regardless of economic interests or intellectual property rights, so the enthusiasm for resource development is not high, which hinders the development of excellent resources.

The information resources such as low-level and repeated-training professional training are common phenomenon in the construction of informationization of vocational education and teaching. In particular, resources such as independent courses and documents have been passively accepted, and lack of interaction and communication [2]. For example, network software, multimedia resources and interactive resources are seriously lacking. Most of the existing information resources only stayed at the low level of being discovered and accepted, and lacked the deeply integrated curriculum part.

\subsection{Incomplete information management}

Management informatization is a process that changes from the traditional management model in the industrial era to the modern management model in the information age. It is based on the collection, processing, and transmission of information resources. It is based on information technology to improve management efficiency. The basic value guideline management is optimization process [2]. The information management of colleges and universities is not simply to electronicize the original business process of the work. It is based on modern management thinking to optimize existing business processes, simplistic process management, rapid information dissemination, and crises-crossing information flows. Information is used as a means to improve management efficiency in colleges and universities.

The level of informatization of administrative personnel directly affects the level of school management and management modernization. Whether or not the college's administrative information construction work can be done well, the human factor is of utmost importance. Their knowledge and understanding of information construction, and their mastery of information technology capabilities directly affect the use and popularization of information technology in teaching [1]. At present, many college administrators lack macroscopic ideas for management informationization, and for those business processes that can use information methods to solve the problem, there is no relatively unified understanding. At the same time, there is a lack of communication and redundant construction of the business systems between departments.

\section{The design and implementation of the construction of smart campus}

When constructing a smart campus, any college should not abandon the existing information equipment. Instead, it should make full use of virtualization technology to fully integrate the existing basic resources to form a fully virtualized resource pool. Logic resources are provided to each application at the same time according to actual needs. By using computer systems, servers and other hardware systems with different architectures to build a comprehensive virtualized application system, and then using heterogeneous storage networks, the computing virtualization clusters can form larger resource pools, realize unified and flexible deployment, and realize services. Smooth migration [3]. This not only can effectively suppress the increase in the number of servers, simplifies the management of server programs, but also significantly improves the utilization of campus servers, network resources, flexibility and reliability.

\subsection{Design of smart campus}

Infrastructure construction mainly includes computer networks, internet of things, and radio and television networks.

1) Construction of computer networks. At present, the construction of computer wired network and wireless network in School A has been initially completed and covers the entire campus. Further construction is required. Build a computer network intranet on the basis of the original computer 
extranet, and use wired and wireless networks [3]. The network covers the entire campus; it is used for the campus multimedia, teaching, logistics and asset management services, dining table supermarket consumption, book lending, dormitory access control, and bathroom and dormitory water control services; Health services network for hospital students and teachers related to medical information management and services.

2) Construction of Internet of Things. In the era of rapid development of the Internet of Things, the construction of a smart campus is possible. In the process of building a smart campus, various information sensing devices will be fully configured and utilized, including radio frequency identifiers, infrared sensors, GPS, and laser scanning devices [3]. Centralized management and control and full use of the internet of things will cover the campus core buildings and key public areas, satisfying the perception, capture, and transfer of relevant people at any time and place. Equipment and resource is information requirements.

3) Radio and television network construction. Campus radio network is divided into indoor and outdoor. Usually used for broadcasting vocals, background music and campus culture propaganda should also cooperate with the fire protection system [3]. In the event of a fire or other emergency, the forced switching is incorporated into the fire radio system to be used as command and dispatch personnel for emergency evacuation. The cable television system should be able to receive local cable television programs and support the use of self-run programs to provide information for multimedia presentations and other applications.

\subsection{Data support platform construction based on Cloud computing}

The cloud-based data support platform is a data platform that supports the efficient operation of smart campuses. It not only requires the centralized storage of basic data, related business information, and teaching resources across the campus, but also requires virtualization and other technologies to realize assets. Centralized management control and flexible allocation is providing resource sharing services. After the completion of the cloud computing center, the teachers and students of the school can easily lease private storage space on the smart cloud service platform to realize the preservation of personal data and other services [4]. Users in various departments and administrative departments can also use virtual hosts on the platform to establish their own websites, scientific research platforms and other applications without having to consider purchasing hardware devices, and more do not need protection and maintenance of the system environment.

\section{Design and Implementation Plan for the Construction of a Smart Campus}

The intensive and intelligent campus is based on information technology, guided by Internet thinking, modern educational theory and humanistic learning theory, and makes full use of new technologies such as mobile internet, cloud computing, big data and internet of things to realize modern technology and modernization. The integration of education and the harmony between people and technology are the main goals, and a safe, stable, environmentally-friendly, energy-saving, and highly efficient smart campus system with the core of "integrated, collaborative development" is being built.

\subsection{Information infrastructure construction}

Infrastructure is the fundamental guarantee for achieving campus intelligence. Its main facilities include information infrastructure based on computer networks, network information services, smart classrooms, simulation training system environments, and digital security systems. Information infrastructure includes campus networks and data centers [4].

1) Campus network reconstruction and expansion. Campus network construction in higher vocational colleges should be a practical, high-speed, stable and reliable and safe and controllable campus network that can be used for school resource sharing, education and teaching, vocational training, and school management. Campus information applications and services, such as online cultural life, provide a network support environment that meets the quality of service requirements.

Combining with the actual situation of the school, according to moderate advances and regulatory 
requirements, we redesigned and constructed the weak electricity pipe network of the whole school and built a new network center room, as shown in Figure 1. Implement wired and wireless network full-coverage projects, build a visual operation and maintenance platform, expand data center, and improve network security protection capabilities.

The network core equipment adopts routers and core switches that support network virtualization. In the initial deployment, the idea of unified planning and step-by-step implementation can be constructed. In the initial period of small business, when the demand is small, a single router and a single core switch can be used to complete the service processing [4]. In the later period, multiple network core devices can be virtually deployed into a single device to be flexibly deployed, and construction can be performed on an as needed basis. At the same time as capital, prevent the waste of capital investment.

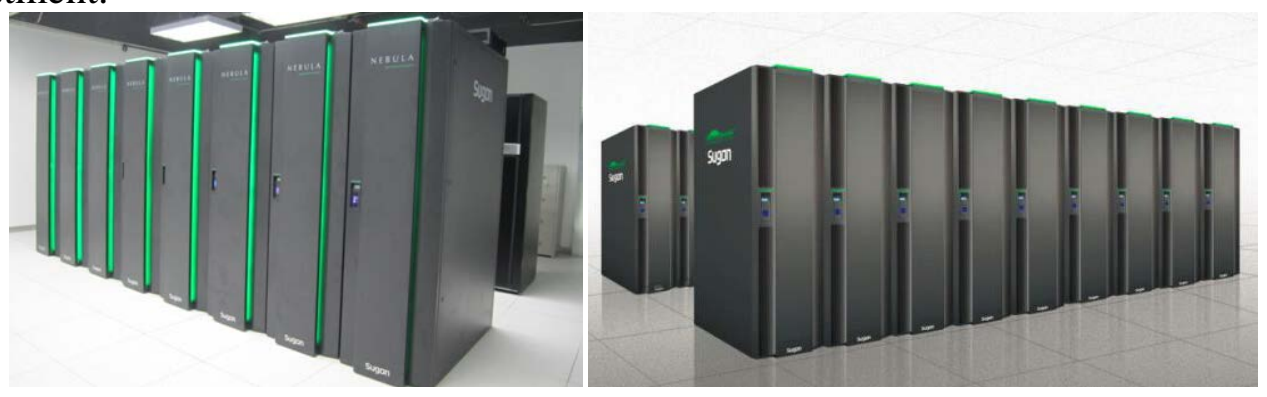

Fig. 1 Standardization of computer room construction

2) Digital Security System Construction: The campus network is used as a transmission platform to build a digital security system based on the IP protocol to realize campus video surveillance, intrusion alarm, access control, electronic patrol, electronic invigilation, fire alarm, emergency call alarm, and emergency The unified management and control of the broadcasting system form an all-dimensional, three-dimensional campus security system, and is networked with the local security department's security system as required. During the construction period, a digital security system covering all sites in the school's old and new campuses will be completed.

3) Construction of the system of the Big One Card. In accordance with the requirements of "One Card at Hand and Smooth Implementation of Campus", we will build a campus card system. The campus ID card service integrates campus user identity identification, school microfinance settlement, school management, financial services, access control management, and book lending. It provides an interface for the school's potential informatization applications or integrates interfaces to support school characteristics.

The campus card hardware system consists of a card data center, front-end system, application subsystem hardware and support software, front-end equipment and card media, card network and other auxiliary equipment.

\subsection{Education and teaching information construction}

The main contents of the information construction of school education and education include the construction of online learning space, digital resource construction, real-time teaching system in the workshop, and the construction of education and teaching management systems [5]. Through the above-mentioned system construction, the methods of school teaching and learning will be changed in an all-round way. Technology is symbiotic, serving teacher and student development.

Digital resources mainly include open resources, introduced resources, and school-based resources. According to the actual situation of the school, it can be used in classrooms and training rooms, digital stadiums and digital libraries [5]. The digital teaching resources used in classrooms and training rooms include general resources and simulation training resources.

A number of "interactive multimedia classrooms", "recorded multimedia classrooms" and "micro-classrooms" were built on the basis of the existing "demonstration-based multimedia classrooms."

Through the construction of interactive multimedia classrooms, face-to-face bi-directional or multi-directional interactive teaching between teachers and students is supported, procedural 
management of assessment and evaluation of teaching and learning, and distance-based teaching based on the Internet are supported.

By building a recording and multimedia classroom, it fully supports the recording of classroom teaching scenes and processes, and supports live classroom teaching on the Internet.

Through the construction of micro-classrooms, it provides independent teachers with a self-training environment for teaching skills, provides an independent training environment for old teachers to learn new skills, reform teaching methods, and teaching models [5]. It also provides independent and part-time teachers with independent recordings of micro lessons, tuition, and flipping. Classroom and other video resources provide a recording and editing environment to accelerate the college's digital resources construction, as shown in Fig. 2.

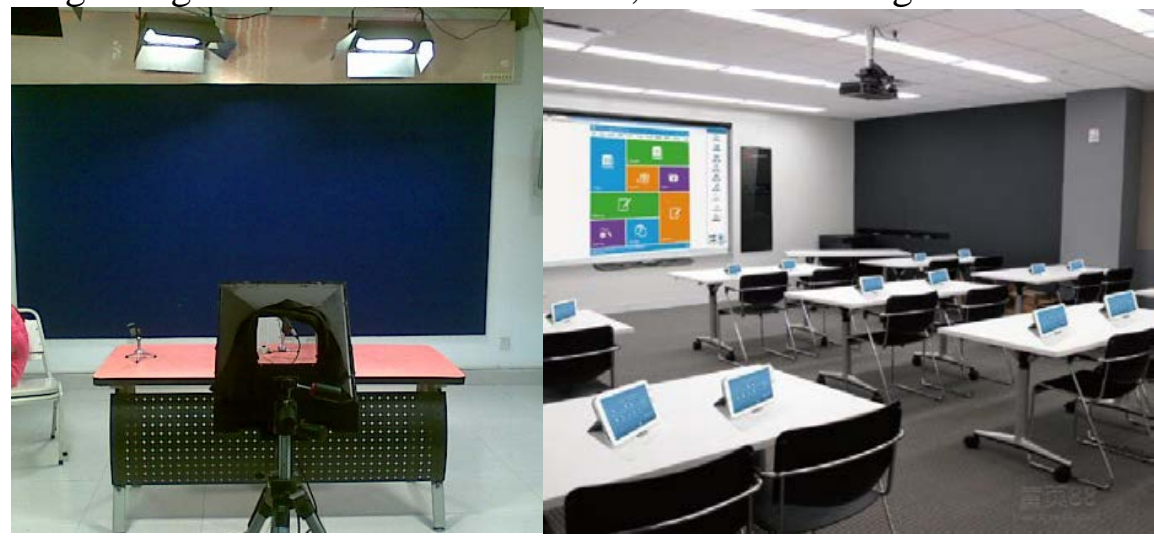

Fig. 2 recording classroom

\section{Summary}

Education informatization is one of the core competencies of domestic and foreign school education. With the rapid development of science and technology, it is a trend and trend for major universities to move from the construction of digital campuses to smart campuses. However, building a smart campus is a very important and long-term project. It needs to be incorporated into the overall development strategy of the school, and a practical and scalable construction plan should be formulated so that the smart campus can truly serve as teachers and students, teaching, scientific research and daily management services.

\section{Acknowledgement}

Fund project: Wuhan city Colleges and universities production research project of 2016 Wuhan Education Bureau. (Project ID: CXY201638).

\section{References}

[1] X.J. Shen and G.H. Gao, Exploration of the Informatization Construction Plan of the Fourth Military Medical University New Campus for Smart Campus, Journal of East China Normal University, 2015, vol.3, pp.12-17.

[2] Y.R. Chen, Research on smart campus system and operation mode based on context awareness, Beijing: Beijing Jiaotong University, 2013, pp.33-35.

[3] D.X. Jiang, Discussion on the Construction of Smart Campus in Universities under the Background of Big Data, Journal of East China Normal University (Natural Science Edition), 2015, vol.3, pp.119-125.

[4] W.W. Yang, Key Problems and Countermeasures Construction of Smart Campus in Colleges and Universities, China Education Informatization, 2013, vol.2, pp.39-42.

[5] Yang Gaodong, The Construction of Smart Campuses in Colleges and Universities, China Education Informatization, 2014, vol.3, pp.21-24. 